\title{
On topological properties of the set of maldistributed sequences
}

\author{
József Bukor \\ Department of Informatics, \\ J. Selye University, \\ Komárno, Slovakia \\ email: bukorj@ujs.sk
}

\author{
János T. Tóth \\ Department of Mathematics, \\ J. Selye University, \\ Komárno, Slovakia \\ email: tothj@ujs.sk
}

\begin{abstract}
The real sequence $\left(x_{n}\right)$ is maldistributed if for any non-empty interval I, the set $\left\{n \in \mathbb{N}: x_{n} \in I\right\}$ has upper asymptotic density 1 . The main result of this note is that the set of all maldistributed real sequences is a residual set in the set of all real sequences (i.e., the maldistribution is a typical property in the sense of Baire categories). We also generalize this result.
\end{abstract}

\section{Introduction}

Following the concept of statistical convergence for real sequences, J. A. Fridy [2] introduced the concept of statistical cluster points of a sequence $\left(x_{n}\right)$. A number $\alpha$ is called a statistical cluster point of the sequence $\left(x_{n}\right)$ provided that for every $\varepsilon>0$ the set $\left\{n \in \mathbb{N}:\left|x_{n}-\alpha\right|<\varepsilon\right\}$ has a positive upper asymptotic density.

G. Myerson [7] calls a sequence $\left(x_{n}\right)$ maldistributed if for any non-empty interval I the set $\left\{n \in \mathbb{N}: x_{n} \in I\right\}$ has upper asymptotic density 1 . In [12] the maldistribution property is characterized by one-jump distribution functions. Examples of maldistributed sequences are given in [12] and [3]. Using the idea from [4] (Example VII) for the generalization of the concept of statistical 
convergence, we can extend the maldistribution property of sequences with the help of weighted densities.

The concept of weighted density as a generalization of asymptotic density was introduced in [1] and [10]. Let $f: \mathbb{N} \rightarrow(0, \infty)$ be a weight function with the properties

$$
\sum_{n=1}^{\infty} f(n)=\infty, \quad \lim _{n \rightarrow \infty} \frac{f(n)}{\sum_{a \leq n} f(a)}=0 .
$$

For $A \subset \mathbb{N}$ define by

$$
\underline{d}_{f}(A)=\liminf _{n \rightarrow \infty} \frac{\sum_{a \leq n, a \in A} f(a)}{\sum_{a \leq n} f(a)} \quad \text { and } \quad \bar{d}_{f}(A)=\limsup _{n \rightarrow \infty} \frac{\sum_{a \leq n, a \in A} f(a)}{\sum_{a \leq n} f(a)}
$$

the lower and upper $f$-densities of $A$, respectively. Note that the asymptotic densities correspond to $f(n)=1$ and the logarithmic densities to $f(n)=\frac{1}{n}$. It is well-known that each set which has asymptotic density also has the logarithmic one but a set may have a logarithmic density without having an asymptotic one.

The main tool to compare weighted densities is the classical result of C. T. Rajagopal (cf. [9], Theorem 3) which, in terms of weighted densities, says the following.

Let $f, g: \mathbb{N} \rightarrow(0, \infty)$ be weight functions with properties (1). If $\frac{f(n)}{g(n)}$ is decreasing, then for any $\mathrm{A} \subset \mathbb{N}$ we have

$$
\underline{\mathrm{d}}_{g}(A) \leq \underline{\mathrm{d}}_{\mathrm{f}}(A) \leq \overline{\mathrm{d}}_{\mathrm{f}}(A) \leq \overline{\mathrm{d}}_{\mathrm{g}}(A) .
$$

Now we give a generalization of maldistributed sequences.

Definition 1 Let $f: \mathbb{N} \rightarrow(0, \infty)$ be a weight function with properties (1). The sequence $\left(\mathrm{x}_{\mathrm{n}}\right)$ is said to be $\mathrm{f}$-maldistributed, if for any non-empty interval I the set $\left\{\mathrm{n} \in \mathbb{N}: \mathrm{x}_{\mathrm{n}} \in \mathrm{I}\right\}$ has upper $\mathrm{f}$-density 1 .

Comparing to asymptotic density, logarithmic density is less sensitive to certain perturbations. For example, if a sequence is maldistributed, then it is not necessary $f$-maldistributed for $f(n)=\frac{1}{n}$ (which defines the logarithmic density). 
Let us denote by $\mathcal{M}_{\mathrm{f}}$ the set of all $\mathrm{f}$-maldistributed sequences. The purpose of this note is to show that for any weight function $\mathrm{f}$ satisfying (1) the set $\mathcal{M}_{\mathrm{f}}$ is residual in the Fréchet metric space of all real sequences.

Let $\mathbf{s}$ be the Fréchet metric space of all sequences of real numbers with the metric

$$
\rho(\mathbf{x}, \mathbf{y})=\sum_{k=1}^{\infty} \frac{1}{2^{k}} \frac{\left|x_{k}-y_{k}\right|}{1+\left|x_{k}-y_{k}\right|},
$$

where $\mathbf{x}=\left(x_{k}\right), \mathbf{y}=\left(y_{k}\right)$. It is known that $(\mathbf{s}, \rho)$ is a complete metric space.

In [5] it was proved that the set of all uniformly distributed sequences is a dense subset of the first Baire category in $\mathbf{s}$. The same is true for the set of all statistically convergent sequences of real numbers (cf. [11]).

\section{Main results}

The main result of this paper is as follows.

Theorem 1 Let $\mathrm{f}: \mathbb{N} \rightarrow(0, \infty)$ be a weight function with properties (1). Then the set of all $\mathrm{f}$-maildistributed sequences $\mathcal{M}_{\mathrm{f}}$ is residual in the the Fréchet metric space of all sequences of real numbers $\mathbf{s}$.

For the proof of the theorem we shall use the following lemma.

Lemma 1 For the interval $\mathrm{I}=[\mathrm{a}, \mathrm{b}]$ denote by $\mathcal{A}(\mathrm{I}, \alpha)$ the set of all $\mathbf{x}=$ $\left(x_{k}\right) \in \mathbf{s}$ for which

$$
\overline{\mathrm{d}}_{\mathrm{f}}\left(\left\{\mathrm{n} \in \mathbb{N}: \mathrm{x}_{\mathrm{n}} \in \mathrm{I}\right\}\right) \leq \alpha,
$$

where $\alpha \in(0,1)$. Then $\mathcal{A}(\mathrm{I}, \alpha)$ is a set of the first Baire category in $\mathbf{s}$.

Proof of Lemma 1. We define a continuous function $h: \mathbb{R} \rightarrow[0,1]$ by

$$
h(x)=\left\{\begin{array}{cll}
\frac{2 x-2 a}{b-a} & \text { for } & x \in\left[a, \frac{a+b}{2}\right] \\
\frac{2 b-2 x}{b-a} & \text { for } & x \in\left[\frac{a+b}{2}, b\right] \\
0 & \text { for } & x \in \mathbb{R} \backslash[a, b]
\end{array}\right.
$$

We choose an arbitrary real number $\beta \in(\alpha, 1)$. Using the function $h$ we define for $\mathbf{x}=\left(x_{k}\right) \in \mathbf{s}$ and fixed $n$ the function $g_{n}: s \rightarrow[0,1]$ in the following way:

$$
g_{n}(x)=\max \left\{\beta, \frac{\sum_{k=1}^{n} h\left(x_{k}\right) \cdot f(k)}{\sum_{k=1}^{n} f(k)}\right\} .
$$


Denote $\mathcal{A}^{*}(\mathrm{I}, \alpha)$ the set of all $\mathbf{x}=\left(x_{k}\right) \in \mathbf{s}$ for which there exists the limit $\lim _{n \rightarrow \infty} g_{n}(\mathbf{x})$.

One can easily check that for each $\mathbf{x}=\left(x_{k}\right) \in \mathbf{s}$ and natural number $\boldsymbol{n}$ we have

$$
\frac{\sum_{k=1}^{n} h\left(x_{k}\right) \cdot f(k)}{\sum_{k=1}^{n} f(k)} \leq \frac{\sum_{k \leq n, x_{k} \in I} f(k)}{\sum_{k \leq n} f(k)} .
$$

For any $\mathbf{x} \in \mathcal{A}(\mathrm{I}, \alpha)$, the right hand side of (3) does not exceed $\alpha$ if $\mathrm{n}$ is large enough. Therefore $\lim _{\mathrm{n} \rightarrow \infty} \mathrm{g}_{\mathrm{n}}(\mathrm{x})=\beta$, and then $\mathcal{A}(\mathrm{I}, \alpha) \subset \mathcal{A}^{*}(\mathrm{I}, \alpha)$.

Put $g(\mathbf{x})=\lim _{n \rightarrow \infty} g_{n}(\mathbf{x})$ for $\mathbf{x} \in \mathcal{A}^{*}(\mathrm{I}, \alpha)$. We shall prove that

(a) the function $g_{n}(n=1,2, \ldots)$ is a continuous function on $\mathbf{s}$,

(b) $\mathrm{g}$ is discontinuous at each point of $\mathcal{A}^{*}(\mathrm{I}, \alpha)$.

(a) Let $\mathbf{x}^{0}=\left(x_{k}^{0}\right)_{k=1}^{\infty}, \mathbf{x}^{(j)}=\left(x_{k}^{(j)}\right)_{k=1}^{\infty} \in \mathbf{s} \quad(j=1,2, \ldots)$ and $\mathbf{x}^{(j)} \rightarrow \mathbf{x}^{0} \quad$ (for $j \rightarrow \infty)$.

Then from the convergence in the space $\mathbf{s}$ for each fixed $k$ we have $\lim _{j \rightarrow \infty} x_{k}^{(j)}=$ $x_{k}^{0}$. The continuity of function $h$ implies $\lim _{j \rightarrow \infty} g_{n}\left(x^{(j)}\right)=g_{n}\left(x^{0}\right)$. Thus $g_{n}$ $(\mathrm{n}=1,2, \ldots)$ is continuous on $\mathbf{s}$.

(b) Let $\mathbf{y}=\left(y_{k}\right) \in \mathcal{A}^{*}(I, \alpha)$. We have the following two possibilities.

(1) $\mathrm{g}(\mathbf{y})<1$,

(2) $\mathrm{g}(\mathbf{y})=1$.

In case (1) we choose a positive $\varepsilon$ such that $\varepsilon<1-\mathrm{g}(\mathbf{y})$. It is suffice to prove that in each ball $\mathrm{K}(\mathbf{y}, \delta)=\left\{\mathbf{x} \in \mathcal{A}^{*}(\mathrm{I}, \alpha), \rho(\mathbf{x}, \mathbf{y})<\delta\right\} \quad(\delta>0)$ of the subspace $\mathcal{A}^{*}(\mathrm{I}, \alpha)$ of $\mathbf{s}$ there exists an element $\mathbf{x}=\left(x_{k}\right) \in \mathbf{s}$ with $\mathrm{g}(\mathbf{x})-\mathrm{g}(\mathbf{y})>\varepsilon$.

Let $\delta>0$. Choose a positive integer $m$ such that $\sum_{k=m+1}^{\infty} 2^{-k}<\delta$, and define the sequence $\mathbf{x}=\left(x_{k}\right)$ in the following way:

$$
x_{k}= \begin{cases}y_{k}, & \text { if } k \leq m, \\ \frac{a+b}{2}, & \text { if } k>m .\end{cases}
$$

Hence $\rho(\mathbf{x}, \mathbf{y})<\delta$, further $h\left(x_{k}\right)=1$ for $k>m$. Then

$$
\frac{\sum_{k=1}^{n} h\left(x_{k}\right) \cdot f(k)}{\sum_{k=1}^{n} f(k)} \geq \frac{\sum_{k=m+1}^{n} f(k)}{\sum_{k=1}^{n} f(k)}=1-\frac{\sum_{k=1}^{m} f(k)}{\sum_{k=1}^{n} f(k)} \rightarrow 1 \text { for } n \rightarrow \infty,
$$


and therefore $g(\mathbf{x})=\lim _{n \rightarrow \infty} g_{n}(\mathbf{x})=1$. Then immediately follows

$$
g(\mathbf{x})-g(\mathbf{y})=1-g(\mathbf{y})>\varepsilon .
$$

In case (2) we have $\mathbf{g}(\mathbf{y})=1$. Let $\delta, \boldsymbol{m}, \mathbf{x}$ have the previous meaning. Put

$$
x_{k}= \begin{cases}y_{k}, & \text { if } k \leq m, \\ a, & \text { if } k>m .\end{cases}
$$

Then, clearly $\rho(\mathbf{x}, \mathbf{y})<\delta$, and $\mathrm{h}\left(\mathrm{x}_{\mathrm{k}}\right)=0$ for $\mathrm{k}>\mathrm{m}$. Then

$$
\frac{\sum_{k=1}^{n} h\left(x_{k}\right) \cdot f(k)}{\sum_{k=1}^{n} f(k)} \leq \frac{\sum_{k=1}^{m} f(k)}{\sum_{k=1}^{n} f(k)} \rightarrow 0 \text { for } n \rightarrow \infty .
$$

So, we have $g(\mathbf{x})=\lim _{n \rightarrow \infty} g_{n}(\mathbf{x})=\beta$, and therefore $g(\mathbf{y})-g(\mathbf{x})=1-\beta>0$. Hence the discontinuity of $\mathrm{g}$ at $\mathbf{y} \in \mathcal{A}^{*}(\mathrm{I}, \alpha)$ has been proved.

The function $\mathrm{g}$ is a limit function (on $\mathcal{A}^{*}(\mathrm{I}, \alpha)$ ) of the sequence of continuous functions $\left(g_{n}\right)_{n=1}^{\infty}$ on $\mathcal{A}^{*}(I, \alpha)$. Then the function $g$ is a function in the first Baire class on $\mathcal{A}^{*}(\mathrm{I}, \alpha)$. According to the well-known fact that the set of discontinuity points of an arbitrary function of the first Baire class is a set of the first Baire category (cf. [8], p. 32), we see that the set $\mathcal{A}^{*}(\mathrm{I}, \alpha)$ is of the first Baire category in $\mathcal{A}^{*}(\mathrm{I}, \alpha)$ Thus $\mathcal{A}^{*}(\mathrm{I}, \alpha)$ is in $\mathbf{s}$, too. Since $\mathcal{A}(\mathrm{I}, \alpha) \subset \mathcal{A}^{*}(\mathrm{I}, \alpha)$, the assertion follows.

Proof of Theorem 1. Denote by $\mathbb{Q}$ the set of all rational numbers. Denote by $\mathcal{H}$ the set of all $\mathbf{x}=\left(x_{k}\right) \in \mathbf{s}$ for which there exists an interval I with

$$
\overline{\mathrm{d}}_{\mathrm{f}}\left(\left\{\mathrm{n} \in \mathbb{N}: \mathrm{x}_{\mathrm{n}} \in \mathrm{I}\right\}\right) \leq \alpha
$$

for some $\alpha \in(0,1)$. Combining Lemma 1 and the fact that for each interval I there exist rational numbers $a, b$ such that $I \subset[a, b]$, we have

$$
\mathcal{H} \subset \bigcup_{a, b \in \mathbb{Q}, a<b} \bigcup_{i \in \mathbb{N}, i \geq 2} A\left([a, b], 1-\frac{1}{i}\right)
$$

from which follows at once that $\mathcal{H}$ is a meager set. But $\mathcal{M}_{\mathrm{f}}=\mathbf{s} \backslash \mathcal{H}$ and therefore the assertion of theorem follows. Hence the property of $f$-maldistribution is a typical property of real sequences from the topological point of view.

We now introduce the concept of $f$-maldistributed integer sequences. 
Definition 2 Let $\mathrm{f}: \mathbb{N} \rightarrow(0, \infty)$ be a weight function with properties (1). The sequence $\left(\mathrm{x}_{\mathrm{n}}\right)$ of positive integers is said to be f-maldistributed, if for any positive integers $m \geq 2$ and $j \in\{0,1, \ldots, m-1\}$ the set $\left\{n \in \mathbb{N}: x_{n} \equiv\right.$ $\mathrm{j}(\bmod \mathrm{m})\}$ has upper $\mathrm{f}$-density 1 .

Let $\mathbf{S}$ be the Baire's space of all sequences of positive integers with the metric $\rho^{\prime}$ defined in the following way.

Let $\mathbf{x}=\left(x_{k}\right) \in \mathbf{S}$, and $\mathbf{y}=\left(y_{k}\right) \in \mathbf{S}$. If $\mathbf{x}=\mathbf{y}$, then $\rho^{\prime}(\mathbf{x}, \mathbf{y})=0$, otherwise

$$
\rho^{\prime}(\mathbf{x}, \mathbf{y})=\frac{1}{\min \left\{n: x_{n} \neq y_{n}\right\}} .
$$

The space $\left(\mathbf{S}, \rho^{\prime}\right)$ is a complete metric space. In [6] the topological properties of the set of all uniformly distributed sequences of positive integers in Baire's space were investigated.

The following auxilary result is similar to Lemma 1.

Lemma 2 For the positive integers $m \geq 2$ and $j \in\{0,1, \ldots, m-1\}$ denote by $\mathcal{A}(\mathbf{j}, \mathrm{m}, \alpha)$ the set of all $\mathbf{x}=\left(\mathrm{x}_{\mathrm{k}}\right) \in \mathbf{S}$ for which

$$
\overline{\mathrm{d}}_{\mathrm{f}}\left(\left\{\mathrm{n} \in \mathbb{N}: \mathrm{x}_{\mathrm{n}} \equiv \mathrm{j}(\bmod \mathrm{m})\right\}\right) \leq \alpha,
$$

where $\alpha \in(0,1)$. Then $\mathcal{A}(j, m, \alpha)$ is a set of the first Baire category in $\mathbf{S}$.

The proof is analogous to the proof of Lemma 1. The crucial role is played by the function $g_{n}: S \rightarrow[0,1]$ given by

$$
g_{n}(x)=\max \left\{\sqrt{\alpha}, \frac{\sum_{k \leq n} f(k)}{\sum_{k=1}^{n} f(k)}\right\} .
$$

The following theorem says that the set of all $\mathrm{f}$-maldistributed integer sequences form a residual set in Baire's space.

Theorem 2 Let $\mathrm{f}: \mathbb{N} \rightarrow(0, \infty)$ be a weight function with properties (1). Denote by $\mathcal{G}$ the set of all $\mathbf{x}=\left(\mathrm{x}_{\mathrm{k}}\right) \in \mathbf{S}$ for which there exist $\mathrm{m} \geq 2$ and $j \in\{0,1, \ldots, m-1\}$ such that

$$
\overline{\mathrm{d}}_{\mathrm{f}}\left(\left\{\mathrm{n} \in \mathbb{N}: \mathrm{x}_{\mathrm{n}} \equiv \mathrm{j}(\bmod \mathrm{m})\right\}\right) \leq \alpha
$$

for some $\alpha \in(0,1)$. Then $\mathcal{G}$ is a set of the first Baire category in $\mathbf{S}$. 
Proof. Combining Lemma 2 with the fact that

$$
\mathcal{G} \subset \bigcup_{m=2}^{+\infty} \bigcup_{j=0}^{m-1} \bigcup_{i=2}^{+\infty} A\left(j, m, 1-\frac{1}{i}\right)
$$

it immediately follows that $\mathcal{G}$ is a meager set in $\mathbf{S}$.

\section{References}

[1] R. Alexander, Density and multiplicative structure of sets of integers, Acta Arith., 12 (1976), 321-332.

[2] J. A. Fridy, Statistical limit points, Proc. Amer. Math. Soc., 118 (1993), 1187-1192.

[3] P. Kostyrko, M. Mačaj, T. Šalát, O. Strauch, On statistical limit points, Proc. Amer. Math. Soc., 129 (2000), 2647-2654.

[4] P. Kostyrko, M. Mačaj, T. Šalát, O. Strauch, I-convergence and extremal I-limit points, Math. Slovaca, 55 (2005), 443-464.

[5] V. László, T. Šalát, The structure of some sequence spaces, and uniform distribution (mod 1), Periodica Math. Hung., 10 (1979), 89-98.

[6] V. László, T. Šalát, Uniformly distributed sequences of positive integers in Baire's space, Math. Slovaca, 41 (1991), 277-281.

[7] G. Myerson, A sampler of recent developments in the distribution of sequences, Lecture Notes in pure and applied Mathematics, 147 (1993), 163-190.

[8] J. C. Oxtoby. Measure and Category. Graduate texts in Mathematics. Springer, 1980.

[9] C. T. Rajagopal, Some limit theorems, Amer. J. Math., 70 (1948), $157-$ 166.

[10] H. Rohrbach, B. Volkmann, Verallgemeinerte asymptotische Dichten, J. Reine Angew. Math., 194 (1955), 195-209. 
[11] T. Šalát, On statistically convergent sequences of real numbers, Math. Slovaca, 30 (1980), 139-150.

[12] O. Strauch, Uniformly maldistributed sequences in a strict sense, Monatsh. Math., 120 (1995), 153-164.

Received: February 20, 2019 